\title{
Effects of Growth Hormone Treatment in Adults with Prader-Willi Syndrome
}

\author{
M.G. Butler ${ }^{1}$, B.K. Smith ${ }^{2}$, J. Lee ${ }^{3}$, C. Gibson ${ }^{4}$, C. SchmolI ${ }^{5}$, W.V. Moore ${ }^{5}$, and J.E. \\ Donnelly 6 \\ ${ }^{1}$ Departments of Psychiatry \& Behavioral Sciences and Pediatrics, University of Kansas Medical \\ Center, Kansas City \\ ${ }^{2}$ Department of Kinesiology and Health Education, Southern Illinois University, Edwardsville, \\ Illinois \\ ${ }^{3}$ Center for Research Methods and Data Analysis, University of Kansas, Lawrence \\ ${ }^{4}$ General and Geriatric Medicine, University of Kansas Medical Center, Kansas City, KS \\ ${ }^{5}$ Department of Pediatrics, Children's Mercy Hospital, Kansas City, MO \\ ${ }^{6}$ Center for Physical Activity \& Weight Management, University of Kansas, Lawrence
}

\begin{abstract}
Objective-Since limited data exist on adults with Prader-Willi syndrome (PWS) and growth hormone $(\mathrm{GH})$ treatment, we report our experience on the effects of treatment for one year on body composition, physical activity, strength and energy expenditure, diet, general chemistry and endocrine data with quality of life measures.
\end{abstract}

Design-We studied 11 adults with PWS (6F:5M; average age $=32$ yrs) over a 2 year period with GH treatment during the first year only. Electrolytes, IGF-I, glucose, thyroid, insulin, lipids, body composition, physical activity and strength, diet, energy expenditure and quality of life data were collected and analyzed statistically using linear modeling at baseline, at 12 months following GH therapy and at 24 months after treatment cessation for 12 months.

Results-Total lean muscle mass was significantly increased $(\mathrm{p}<0.05)$ during GH treatment along with moderate-vigorous physical activity and plasma IGF-I and HDL levels, but returned to near baseline after treatment. Percent body fat decreased during the 12 months of GH treatment but increased after treatment.

Conclusions-Previously reported beneficial effects of GH treatment in children with PWS were found in our adults regarding body composition, physical activity and plasma HDL and IGFI levels. Several beneficial effects diminished to near baseline after cessation of GH treatment for 12 months supporting the continuation of treatment in PWS into adulthood and possibly adults not previously treated during childhood.

Correspondence to: Merlin G. Butler, MD, PhD, Departments of Psychiatry \& Behavioral Sciences and Pediatrics, Kansas University Medical Center, 3901 Rainbow Blvd, MS 4015, Kansas City, Kansas 66160, Phone: (913) 588-1873, Fax: (913) 588-1305, mbutler4@kumc.edu.

Disclosure Summary: The authors have no conflicts of interest. 


\section{Keywords}

Growth hormone treatment; Prader-Will syndrome adults; body composition; energy expenditure; physical activity

\section{Introduction}

Prader-Willi syndrome (PWS) is a complex genomic imprinting disorder affecting multiple body systems including hypotonia, poor suck and feeding problems, hypogonadism/ hypogenitalism, growth hormone (GH) deficiency with short stature and small hands/feet, hyperphagia and obesity, learning/behavior problems and a characteristic face. ${ }^{1-5} \mathrm{PWS}$ is due to absence of paternally expressed genes in the 15q11-q13 chromosome region from a deletion, maternal disomy 15 or an imprinting defect. ${ }^{2,3,6}$

Children with PWS grow at different rates than other children but most are born with a normal weight and length. Failure to thrive occurs but they gradually gain strength and muscle mass before onset of hyperphagia and obesity in early childhood. The fat mass increases more rapidly than muscle mass or stature in children with PWS without growth hormone treatment with more than twice the amount of body fat gained compared with others and often measuring about $50 \%$ of their total body weight. ${ }^{7}$ Weight control with exercise and dietary restrictions are key management issues. ${ }^{2,8,9}$

Due to growth hormone deficiency reported in PWS, the Food and Drug Administration approved treatment in children genetically confirmed with PWS in 2000 without prior laboratory determination of growth hormone deficiency. GH treatment is now commonly prescribed in infancy and childhood to increase stature in PWS but also increases muscle and decreases fat thereby lowering risk factors for diabetes and cardiopulmonary problems. Increased alertness, physical activity and self-esteem in older children are potential effects of GH treatment ${ }^{10-15}$ which should improve the quality of life applicable in adulthood. In addition, Lindgren and Lindberg ${ }^{16}$ documented that GH treatment in children with PWS appear to normalize to an adult height with improvement in body composition, an important outcome for individuals with this disorder. PWS children appear to be highly sensitive to GH suggesting that growth factors (i.e., insulin-like growth factor I or IGF-I) should be monitored closely (e.g., every three months) and maintained within the normal range for age. There is a paucity of data on the use of GH in adults with PWS not previously treated and related to energy expenditure, physical activity, body composition, endocrine and quality of life measures.

The main objective of our study was to examine how GH treatment for one year would impact on body composition, quality of life, physical activity and energy balance in adults with PWS using novel approaches, and if loss of a positive effect, if present, during therapy disappears after cessation of treatment for 12 months. Anticipated outcomes:

1. Increase IGF-I level thereby lessoning risk factors for co-morbidity; improve quality of life measures; decrease fat, increase lean body mass and bone density determined by DEXA. 
2. Increase energy expenditure as determined by whole-room calorimeter measures, diet records, physical activity monitors, strength and endurance measures.

\section{Materials and Methods}

\section{Subjects}

We screened 12 genetically confirmed Caucasian adults with PWS and enrolled 11 adults $(6 \mathrm{~F}: 5 \mathrm{M}$; average age $\pm \mathrm{SD}=32.3 \mathrm{y} \pm 11.1 \mathrm{y}$; age range $=23 \mathrm{y}$ to $50 \mathrm{y})$ for a non-controlled two year study on the effects of GH treatment for 12 months followed by an observation period of 12 months without treatment. The inclusion criteria for selection were as follows: Sixteen to 60 years of age; male or female with diagnosis of PWS confirmed by genetic testing; low plasma IGF-I level (e.g., $<25 \%$ ile) at baseline; and ability to provide informed consent or availability of a suitable legally authorized representative. The exclusion criteria were as follows: Pregnancy; previous GH treatment; untreated or uncontrolled endocrine disease (e.g., diabetes or thyroid disorder); history of severe scoliosis; uncontrolled high blood pressure, heart disease or history of stroke; weight greater than $95 \%$ ile based on gender and established age specific growth charts for PWS ${ }^{17}$ ) and severe breathing problems by history or sleep apnea found by sleep center polysomnography as a diagnostic tool used in sleep medicine. The adults with PWS enrolled in the study showed evidence of hypogonadism (i.e., males with small, undescended testicles and females with absent or sparse history of menstruation) with low plasma estrogen or testosterone levels.

The subjects were recruited from a multi-disciplinary clinic specializing in PWS directed by one of the authors (MGB) following informed consent with the study protocol approved by the local institutional review board. Height and weight were determined at each visit. Height (measured to the nearest $0.1 \mathrm{~cm}$ using a Forgue electric stadiometer) and weight (measured to the nearest $0.1 \mathrm{~kg}$ using a calibrated electronic scale, height) were obtained by one of the authors (CS). Heights that were discordant from previous measurements were confirmed by repeat measurement. Variations in height were attributed to lack of cooperation by the subjects. Age, weight, height, body mass index $\left(\mathrm{BMI} \mathrm{kg} / \mathrm{m}^{2}\right)$, percent body fat and energy expenditure data are shown in Table 1. Nine PWS adults had the typical 15q11-q13 deletion; one with maternal disomy 15 and one with an imprinting defect determined by genetics testing. ${ }^{4,5}$ During this study, daily subcutaneous injections of growth hormone (e.g., Genentech Nutropin AQ; $0.0125 \mathrm{mg} / \mathrm{kg} /$ day) were administered which were comparable to dosages reported previously in PWS adults and based on the treatment of adult GH deficiencies. ${ }^{18}$ Each subject and care provider underwent training for GH administration, recording of dosage and monitoring for potential side effects (edema, headaches and signs of allergic reactions). Compliance for growth hormone use was determined by monitoring the amount of GH unused in the kit when returned at each scheduled clinic visit (e.g., monthly). At the time of the physical examination and measurements, blood was collected for laboratory studies before a new package of $\mathrm{GH}$ and administration supplies were given to the subject for continuation of treatment. Growth hormone dosages were adjusted in the first 3 months of treatment to achieve plasma IGF-I levels within the therapeutic reference range for at least 9 months during the first year. Each assessment was undertaken over a two year 
period (at baseline, at 12 months of GH therapy and at 24 months or one year following treatment).

\section{Methods}

Fasting Blood Samples and Body Composition Measures-Fasting blood samples were obtained for general chemistry (electrolytes), plasma IGF-I, glucose, thyroid, insulin and lipid levels at screening, baseline, 1 month, 2 months, 3 months, 6 months, 9 months, 12 months and 24 months using established protocols in the Children's Mercy Hospital clinically approved certified laboratories for patient care. Due to hyperphagia and food seeking behavior as major components of PWS, the fasting status of each subject could not be confirmed for all measurements. Dual energy x-ray absorptiometry (DEXA - General Electric Prodigy, Atlanta, GA) was used to determine body composition (lean muscle and fat) and bone mineral density following established protocols. ${ }^{19}$ Provocative growth hormone stimulation testing was not performed prior to enrolling in the study. All subjects were healthy and not undergoing medical care for chronic illnesses related to their disorder.

Dietary Intake-All participants were asked to complete a 3-day food record with the assistance of their care provider in order to determine the average caloric intake. Because of the range of cognitive abilities in individuals with PWS and their food seeking behavior, obtaining accurate measurements of dietary intake would be difficult. Thus, to increase the accuracy of caloric intake, the 3-day food records were sent to the participant as well as the primary caregiver for completion. The primary caregiver was asked to assist each participant in recording all foods consumed during 3 days ( 2 week days and 1 weekend day). Primary caregivers were provided detailed instructions on completion of the 3-day food records and were asked to track all food consumption during the three day periods. To increase accuracy of the records, caregivers were asked to provide labels and/or packaging from all packaged foods. Upon completion, registered dietitians followed up with the participants to ensure the accuracy of the records and reviewed the records for completeness and nutrient calculations performed on the recorded data using the Nutrition Data System for Research Software 2010 (University of Minnesota, Minneapolis, MN).

Strength Index - Voluntary physical activity was measured by CSA accelerometers and strength by the one repetition of maximum method (1RM) with two exercises (bench and leg press) on all subjects following established protocols. ${ }^{20}$ Assessments of muscular strength (assessed by 1RM) were obtained at baseline, at 12 and 24 months using bench press (Paramount Fitness Corporation, Los Angeles, CA) and leg press (Hammer Strength, Shiller Park, IL) according to the protocol described by Lemmer et al. ${ }^{20}$ The participants initially completed a light 5 repetition warm-up period with minimal weight. After a brief rest for 60 seconds, a weight estimated to be less than the participant's 1RM was chosen and the participant was instructed to lift the weight through a full range of motion. If the lift was successful, the weight was increased then followed by a 60 second rest. They were then asked to lift the increased weight again and 1RMs obtained in less than 5 lifts for exercise. This procedure was repeated until a weight was reached that the participant could no longer lift. The maximum weight lifted through the full range of motion was recorded as their $1 \mathrm{RM}$. 
Indirect Calorimeter and Metabolic Measurements-A whole-room indirect calorimeter as described previously ${ }^{19,21}$ was used to continuously measure energy expenditure over an 8 hour period at baseline, and at the end of year one following GH treatment and at year two. Twenty-four hour energy expenditure was then extrapolated from the 8 hour measurement. ${ }^{21}$

The completed 3-day food records were used to develop standardized menus to mimic the participant's habitual diet (kcal and macronutrient composition) while in the whole-room calorimeter. Participants were asked to follow the menus for the 3 day period prior to their baseline metabolic measurement which were used in the whole-room calorimeter. These same menus were then provided to each participant prior to each subsequent visit and they were asked to follow these menus during the 3 days prior to testing.

Participants entered the whole-room calorimeter at 8:00am and remained in the room until 4:00pm. Upon entering the calorimeter, participants were asked to lie quietly, but not sleep, for the first 60 minutes of the whole-room stay in order to measure their resting metabolic rate (RMR). The participants were then allowed to watch TV, read or play games while in the room. They were not allowed to sleep while in the calorimeter. Research staff checked on the participants at least every 30 minutes to ensure that the participants were awake. Standardized meals scheduled to be consumed during the testing period were provided to the participant at the appropriate times.

Urine was collected during the calorimeter stay for the determination of total nitrogen concentration $^{22}$ to assess protein oxidation. ${ }^{23,24}$ Using measured oxygen consumption and carbon dioxide production, respiratory quotient (RQ), energy expenditure and substrate oxidation were calculated using the equations of Jequier et al. ${ }^{25}$

Physical Activity Measurements-Daily physical activity was measured with accelerometers [Actigraph GTM1 (Actigraph, Pensacola, FL)] at baseline and at year one and year two and mailed to the participants with specific instructions on wearing the device approximately 10 days prior to visit. Participants wore the accelerometer on a belt over their non-dominant hip for seven consecutive days prior to their visit except when sleeping, swimming or bathing. Upon arrival to the laboratory, the accelerometers were collected and data downloaded. Data were collected in one-minute epochs and a minimum of 12 hours required to be considered a valid monitored day. Intensity cut-points were based upon the National Health and Nutrition Examination Survey reported by Troiano et al. ${ }^{26}$ with sedentary ( $\leq 99$ counts/minute), light (100 to 2019 counts/minute), moderate (2020 to 5999 counts/minute) and vigorous ( $\geq 6000$ counts $/ \mathrm{min}$ ) recorded on each subject.

Quality of Life Assessments-Two quality of life instruments were selected for administration to the PWS adults. The Quality of Life-Assessment of Growth Hormone Deficiency in Adults (QoL-AGHDA) questionnaire was administered initially to the PWS adults by one of the authors (BKS) by reading questions along with the possible choices to each participant. No additional explanations of the study questions were required for each subject to participate successfully in the assessments. The QoL-AGHDA is a 25-item disease-specific instrument used to assess patients' perceptions about their physical, social, 
and mental health for adults with growth hormone deficiency. ${ }^{27,28} \mathrm{~A}$ total score was produced by summing across all items; a higher QoL-AGHDA score indicates a poorer quality of life. This instrument has been shown to be both reliable and valid with a high level of internal consistency. The short form (SF) - 36 was also used which is a validated self-administered questionnaire that assesses eight health dimensions, including physical functioning, role limitations related to physical functioning, general health perception, vitality, pain, social functioning, emotional well-being and role limitations due to emotional health. ${ }^{29}$ Dimension scores were standardized between 0 and 100, with higher scores representing a better quality of life and previously used on individuals with cognitive impairment without comprising reliability and validity ${ }^{30}$ and also utilized with GH-treated PWS adults. ${ }^{31}$ The SF-36 scale was also administered at baseline, at 12 months following growth hormone therapy and at 24 months after one-year cessation from growth hormone treatment.

\section{Data Analysis}

Our primary endpoint was the longitudinal comparisons of body composition, blood chemistry and physical activity measures between different stages of growth hormone treatment over a 2-year period (baseline, at 12 months while on GH treatment and at 24 months after treatment). We used mixed modeling ${ }^{32}$ to handle non-independence of the data where the outcome measures were collected at multiple time points. The model effects and the error covariance components for the repeated measures were estimated using the restricted maximum likelihood (REML) procedure. In the mixed models, total lean gram, total percent body fat, average counts of moderate-vigorous bouts per day, average length of moderate-vigorous bouts per day, IGF-I, HDL and quality of life scores were contrasted between three measurement periods (baseline, 12 months, 24 months) adjusting for the participants' gender. The compound symmetry error structure provided a smaller AIC [(Akaikes information criteria) or model fit index used to select a model among a finite set of models] and BIC score [(Bayesian information criteria) or another model fit index used for model selection, which introduces a penalty term for the number of parameters in the model] than did the completely general (unstructured), first-order autoregressive and variance component error structures and thus chosen for the model system. All analyses were conducted using SAS 9.231 (SAS Institute, Cary, NC).

\section{Results}

The data were analyzed using mixed modeling to handle interdependency among observations and descriptive characteristics grouped by gender (Table 1). All PWS adults (45\% were male) were Caucasian with an average age $( \pm \mathrm{SD})$ of $32.3 \mathrm{y} \pm 11.1 \mathrm{y}$. Figure 1 presents the adjusted means of each outcome measure at baseline, 12 months and 24 months. In the mixed model system, total lean gram, number of bouts of moderate-vigorous physical activity per day, the average plasma IGF-I levels, which were low or outside the reference range at baseline in our subjects and HDL levels were increased during the growth hormone treatment phase of the study (baseline to 12 months), but after treatment (12 to 24 months) the measures decreased back to baseline levels. The adverse events that might be related to growth hormone therapy included one episode of mild edema of the lower leg in a single 
patient (50 year old male) during the first month of GH treatment which stabilized during the rest of the treatment period and impaired glucose tolerance in four subjects defined as one plasma glucose level over $100 \mathrm{mg} / \mathrm{dl}$. The average pretreatment plasma glucose level in these subjects was $82 \pm 6 \mathrm{mg} / \mathrm{dl}$ before and $99 \pm 22 \mathrm{mg} / \mathrm{dl}$ during $\mathrm{GH}$ treatment. In all instances, the elevated glucose level was sporadic. One individual had a plasma glucose level over $100 \mathrm{mg} / \mathrm{dl}$ (i.e., $102 \mathrm{mg} / \mathrm{dl}$ ) before GH treatment and impaired glucose tolerance noted during treatment. A second individual had a plasma glucose level over $100 \mathrm{mg} / \mathrm{dl}$ (i.e., $102 \mathrm{mg} / \mathrm{dl}$ ) before $\mathrm{GH}$ treatment and normal plasma glucose during treatment. All subjects with impaired glucose tolerance during the treatment phase had normal plasma glucose on follow-up measurements. One individual had well controlled type 2 diabetes and the overall glycemic control did not deteriorate during treatment. The remainder of the subjects had glucose levels less than $100 \mathrm{mg} / \mathrm{dl}$ before, during, and after treatment. The elevated plasma glucose levels did not correlate with the plasma IGF1 levels. The participants lowered their total percent body fat during the first 12 months of GH treatment, but they regained a fat mass more than what they reduced during the 12 months following GH treatment. These changes were significant at 0.05 alpha level, except for the moderate-vigorous physical activity changes from 12 to 24 months and pain and HDL changes from baseline to 12 months. There were no significant differences between time points for diet records, resting metabolic rate, 8 hour energy expenditure or respiratory quotient (Table 2).

\section{Discussion}

Growth hormone replacement in children with PWS has well-defined benefits and risks, although data are limited for adults with PWS. However, adults with PWS share similarities with GH deficient patients (GHD) regarding reduced lean body mass (LBM) and increased fat mass (FM). GH treatment in non-PWS GHD adults improves body composition and has beneficial effects on muscle strength and physical performance. The reports on GH treatment in adults with PWS, to date, indicate improved body composition with increased LBM, a reduction in total body fat, subcutaneous adiposity and visceral fat but with small increases in fasting glucose after 12 months of treatment. ${ }^{33}$ Positive effects of GH treatment on cognitive functioning were noted with improved verbal reasoning and visuospatial skills and motor performance in children. ${ }^{34-36}$

We report a novel approach in PWS by measuring energy balance and comparing data obtained from a monitored whole room calorimeter, daily physical activity measures and quality of life assessments. Quality of life assessments (i.e., SF-36) were recorded in relationship to repeated body composition, blood chemistry and hormone levels, dietary records and strength indices in the selected PWS adults at baseline, after 12 months of GH treatment and at 24 months while off GH treatment for 12 months.

Other GH treatment trials reported in PWS men and women included randomized treatment with GH or a placebo for one year followed for two years with open-labeled GH treatment. ${ }^{37}$ At one year, IGF-I levels were increased by 1.5 fold and body composition improved. We also observed an increase (about 4 fold) over baseline in plasma IGF-I levels after 12 months of GH treatment. Positive effects on body composition (i.e., decreased visceral and thigh fat and increased muscle mass) were reported by Sode-Carlsen et al. ${ }^{37-39}$ 
in their Scandinavian cohort of PWS adults after two years of GH treatment. The beneficial effects occurred without serious adverse events while lipid and glucose metabolism data were unchanged. We also observed the positive impact on body composition with decreased total percent body fat after GH treatment but returned to near baseline after treatment cessation for 12 months. One of our PWS males did experience mild leg edema during treatment. High density lipoprotein levels did increase in our PWS adults during GH treatment but returned to a lower (less beneficial) level after treatment cessation.

Mogul et al. ${ }^{18}$ in 2008 reported the effectiveness and safety of GH in 38 GH-deficient PWS adults with a mean age of 30.5 years over a 12 -month period using both six-month doseoptimization and six-month stable treatment periods. Human recombinant GH was initially administered at $0.2 \mathrm{mg} /$ day with monthly $0.2 \mathrm{mg}$ increments to a maximum of $1.0 \mathrm{mg} / \mathrm{day}$, as tolerated, comparable to the dosage used in our study. They reported that lean body mass increased by about $3 \mathrm{~kg}$ and percent body fat decreased by $3 \%$ in their 30 PWS adults who completed the 6-12 month trial of GH treatment. Mildly progressive ankle edema was the most serious treatment adverse event reported in 5 of their 38 PWS adults. The total lean body mass in our study increased significantly by $2.3 \mathrm{~kg}$ during GH treatment but returned to a near baseline level after cessation of treatment for 12 months. The mean fasting glucose and insulin levels in their study were normal at baseline and remained in the normal reference range during GH treatment which agreed with the results found in our study. Although glucose levels were higher at 12 months of GH treatment in our PWS adults compared to baseline (e.g., $116 \mathrm{vs} 101 \mathrm{mg} / \mathrm{dl}$, respectively), the levels were not significantly different (see Table 2). No PWS adults in our study were diagnosed with diabetes during $\mathrm{GH}$ treatment.

In a separate study, Angulo et al. ${ }^{40}$ in 2007 evaluated the final adult height in children with PWS and with and without GH. They found that administration of growth hormone in infancy and/or early childhood restored linear growth and final adult height without significant adverse events. In contrast, children with PWS who had not received growth hormone treatment had a significant decrease in growth velocity, with a mean final adult height under 2 standard deviations below the relevant population mean and with a higher risk for type 2 diabetes mellitus.

Lastly, endocrine and metabolic aspects of adults with PWS were studied by Hoybye ${ }^{41}$ in 2004 with special emphasis on the effect of growth hormone on a cohort of 19 young adults with clinical PWS having a mean BMI of $35 \mathrm{~kg} / \mathrm{m}^{2}$. At baseline the activity of the GH-IGF-I system was impaired and bone mineral density was low, as similarly seen in our PWS adults. Seventeen adults with PWS subsequently completed 12 months of GH treatment without significant adverse effects. Their study showed that adults with PWS had a partial GH deficiency, and GH treatment showed beneficial effects on body composition in adults with PWS without significant side-effects.

Bertella et al. ${ }^{31}$ reported their experience with quality of life measures in a study of 13 adults with PWS treated with GH using the SF-36 quality of life instrument to assess eight health dimensions. They found significant improvements with GH treatment in physical 
functioning, body pain and general mental health. We also recorded higher scores for 4 of the 8 health dimension variables tested during GH treatment in our PWS study.

In conclusion, our study on GH treatment in adults with PWS of both sexes showed a positive impact on several variables including total lean gram weight, moderate-vigorous bouts of physical activity and plasma IGF-I and HDL levels during the growth hormone treatment interval but fasting glucose levels increased during treatment but not significantly different compared with baseline. The average fasting glucose levels were also high (outside of the normative reference range at 12 months) following $\mathrm{GH}$ treatment. The average baseline insulin levels were also high in comparison to reference ranges for our PWS adults. However, after cessation of GH treatment for 12 months, several variables returned close to baseline levels. In contrast, our PWS adults showed lower total percent body fat after 12 months of growth hormone treatment, but they regained more body fat than what they lost during the 12 months off treatment. All changes were significant at 0.05 alpha levels, except for the number of moderate-vigorous bouts of spontaneous physical activity recorded changes from 12 to 24 months and HDL levels from baseline to 12 months after GH treatment. Considering the extremely low physical activity levels at baseline in the PWS adults, the lack of change in the number of moderate-vigorous bouts of spontaneous physical activity from 12 to 24 months can be viewed as a positive outcome. An increase in moderate-vigorous spontaneous activity, regardless of size, has the potential to have positive long term benefits on weight management and overall health. The quality of life instruments were used to assess PWS adults receiving growth hormone and showed improvement with higher scores recorded for 4 of the 8 variables tested between baseline and at 12 or 24 months implying improvement during GH treatment for emotional and physical health, energy, social and particularly pain sensation. Interestingly, individuals with PWS are known to have decreased pain sensation and the change in pain perception during $\mathrm{GH}$ treatment will require further investigation.

There were no significant differences identified between time point measures for resting metabolic rate, 8 hour energy expenditure, or respiratory quotient using the whole room calorimeter despite an increase in lean body mass from the effects of GH treatment in our study. In the literature, the energy requirement in non-PWS individuals for lean body mass is estimated at about 15 to $25 \mathrm{kcal}$ per kg per day ${ }^{42,43}$ and the lean body mass increased by $2.3 \mathrm{~kg}$ on average in our PWS adults following GH treatment. Therefore, the potential increase in resting metabolic rate would be estimated at 34-57 kcal which would account for little change in the resting metabolic rate detectable in our study. A second potential factor to consider for the lack of difference in energy expenditure measures may be the testing conditions. Although the whole room indirect calorimetry does allow for accurate measurements of energy expenditure, the space restrictions may limit spontaneous physical activity by the subject during the course of the day. Reduced spontaneous physical activity due to space restrictions in the whole room chamber could result in lower 24 hour metabolic rate calculations when compared to free-living arrangements outside of the chamber.

Several beneficial effects of growth hormone treatment noted previously in children with PWS were also observed in our PWS adults, specifically improved body composition, increased physical activity (number and duration of spontaneous bouts of activity or 
movement) and higher morning fasting plasma HDL (12 months compared with 24 months) and IGF-I levels. Several of the measures appeared to regress to baseline after GH treatment cessation for 12 months. Due to better recognition, care and treatment including prevention of extreme obesity and co-morbidities, it is expected that GH treatment in PWS adults may exceed 50 years as affected patients are now living to an advanced age. Our study is not definitive due to sample size and length of treatment but supports the accumulating data on the use of GH in treating PWS adults with or without prior GH therapy to produce beneficial effects on body composition, physical activity, lipid profiles and possibly on quality of life with minimal adverse effects but glucose and insulin levels should be followed during GH treatment as recommended in treating non-PWS GH deficient patients. The authors do encourage an expanded study with a larger group of patients including those at an older age and obtain long term outcome data greater than two years duration on potential side effects (e.g., increased glucose levels, insulin resistance, edema) before a firm decision can be made regarding use of long term GH treatment and cost effectiveness in PWS adults. The implications and cost of long term GH treatment to each individual and to society and added health risks will warrant more investigation and discussion.

\section{Acknowledgments}

We thank the adults with Prader-Willi syndrome and their families who participated in the study. The study was partially supported by Genentech, Inc. who also provided the growth hormone and supplies. We thank Carla Meister for expert preparation of the manuscript.

\section{References}

1. Butler MG. Prader-Willi syndrome: current understanding of cause and diagnosis. Am. J. of Med. Genet. 2009; 35:319-332. [PubMed: 2309779]

2. Butler, MG.; Lee, PDK.; Whitman, BY. Management of Prader-Willi Syndrome. 3rd edition. New York: Springer-Verlag; 2006. p. 1-550.

3. Cassidy SB, Driscoll DJ. Prader-Willi syndrome. Eur. J. Hum. Genet. 2009; 17:3-13. [PubMed: 18781185]

4. Butler MG. Prader-Willi syndrome: obesity due to genomic imprinting. Curr. Genomics. 2011; 12:203-223.

5. Cataletto M, Angulo M, Hertz G, Whitman B. Prader-Willi syndrome: A primer for clinicians. Int. J. Pediatr. Endocrinol. 2011; 1:12. [PubMed: 22008714]

6. Bittel DC, Butler MG MG. Prader-Willi syndrome: clinical genetics, cytogenetics and molecular biology. Expert Rev. Mol. Med. 2005; 7:1-20. [PubMed: 16038620]

7. Meaney FJ, Butler MG. The developing role of anthropologists in medical genetics: anthropometric assessment of the Prader-Labhart-Willi syndrome as an illustration. Med. Anthropol. 1989; 10:247253. [PubMed: 2747454]

8. Goldstone AP, Holland AJ, Hauffa BP, et al. Recommendations for the diagnosis and management of Prader-Willi syndrome. J. Clin. Endocrinol. Metab. 2008; 93:4183-4197. [PubMed: 18697869]

9. McCandless SE. Clinical report-health supervision for children with Prader-Willi syndrome. Pediatr. $2011 ; 127: 195-204$.

10. Myers SE, Carrel AL, Whitman BY, et al. Sustained benefit after 2 years of growth hormone on body composition, fat utilization, physical strength and agility, and growth in Prader-Willi syndrome. J. Pediatr. 2000; 137:42-49. [PubMed: 10891820]

11. Haqq AM, Stadler DD, Jackson RH, et al. Effects of growth hormone on pulmonary function, sleep quality, behavior, cognition, growth velocity, body composition, and resting energy expenditure in Prader-Willi syndrome. J. Clin. Endocrinol. Metab. 2003; 88:2206-2212. [PubMed: 12727976] 
12. Lindgren AC, Hagenas L, Muller J, et al. Growth hormone treatment of children with Prader-Willi syndrome affects linear growth and body composition favourably. Acta Paediatrica. 1998; 87:2831. [PubMed: 9510443]

13. Eiholzer U, Gisin R, Weinmann C, et al. Treatment with human growth hormone in patients with Prader-Labhart-Willi syndrome reduces body fat and increases muscle mass and physical performance. Eur. J. Pediatr. 1998; 157:368-377. [PubMed: 9625332]

14. Carrel AL, Myers SE, Whitman BY, et al. Growth hormone improves body composition, fat utilization, physical strength and agility, and growth in Prader-Willi syndrome: a controlled study. J. Pediatr. 1999; 134:215-221. [PubMed: 9931532]

15. Carrel AL, Moerchen V, Myers SE, et al. Growth hormone improves mobility and body composition in infants and toddlers with Prader-Willi syndrome. J. Pediatr. 2004; 145:744-749. [PubMed: 15580194]

16. Lindgren AC, Lindberg A A. Growth hormone treatment completely normalizes adult height and improves body composition in Prader-Willi syndrome: experience from KIGS (Pfizer International Growth Database). Horm. Res. 2008; 70:182-187. [PubMed: 18663319]

17. Mogul HR, Lee PD, Whitman BY, et al. Growth hormone treatment of adults with Prader-Willi syndrome and growth hormone deficiency improves lean body mass, fractional body fat, and serum triiodothyronine without glucose impairment: results from the United States multicenter trial. J. Clin. Endocrinol. Metab. 2008; 93:1238-1245. [PubMed: 18211968]

18. Butler MG, Meaney FJ. Standards for selected anthropometric measurements in Prader-Willi syndrome. Pediatrics. 1991; 88:853-860. [PubMed: 1896298]

19. Butler MG, Theodoro MF, Bittel DC, et al. Energy expenditure and physical activity in PraderWilli syndrome: comparison with obese subjects. Am. J. Med. Genet. A. 2007; 143:449-459. [PubMed: 17103434]

20. Lemmer JT, Ivey FM, Ryan AS, et al. Effect of strength training on resting metabolic rate and physical activity: age and gender comparisons. Med. Sci. Sports Exerc. 2001; 33:532-541. [PubMed: 11283427]

21. Chen KY, Sun MG M, et al. Development and validation of a measurement system for assessment of energy expenditure and physical activity in Prader-Willi syndrome. Obes. Res. 1999; 7:387394. [PubMed: 10440595]

22. Kirk EP, Donnelly JE, Smith BK, et al. Minimal resistance training improves daily energy expenditure and fat oxidation. Med. Sci. Sports Exerc. 2009; 41:1122-1129. [PubMed: 19346974]

23. Skogerboe KL, Labbe RF, Rettmer RL, et al. Chemiluminescent measurement of total urinary nitrogen for accurate calculation of nitrogen balance. Clin. Chem. 1990; 36:752-755. [PubMed: 2337984]

24. Livesey G, Elia M. Estimation of energy expenditure, net carbohydrate utilization, and net fat oxidation and synthesis by indirect calorimetry: evaluation of errors with special reference to the detailed composition of fuels. Am. J. Clin. Nutr. 1988; 47:608-628. [PubMed: 3281434]

25. Jequier E, Acheson K, Schutz Y. Assessment of energy expenditure and fuel utilization. Ann. Rev. Nutr. 1987; 7:187-208. [PubMed: 3300732]

26. Troiano RP, Berrigan D, Dodd KW, et al. Physical activity in the United States measured by accelerometer. Med. Sci. Sports Exerc. 2008; 40:181-188. [PubMed: 18091006]

27. McKenna SP. The QoL-AGHDA: an instrument for the assessment of quality of life in adults with growth hormone deficiency. Qual. Life Res. 1999; 8:373-383. [PubMed: 10472170]

28. Wiren L, Whalley D, McKenna S, et al. Application of a disease-specific, quality-of-life measure (QoL-AGHDA) in growth hormone-deficient adults and a random population sample in Sweden: validation of the measure by rasch analysis. Clin. Endocrinol. 2000; 52:143-152.

29. Jenkinson C, Coulter A, Wright L L. Short form 36 (SF36) health survey questionnaire: normative data for adults of working age. BMJ. 1993; 306:1437-1440. [PubMed: 8518639]

30. Baumstarck K, Pelletier J, Aghababian V, et al. Is the concept of quality of life relevant for multiple sclerosis patients with cognitive impairment? Preliminary results of a cross-sectional study. PLoS ONE. 2011; 7:e30627. [PubMed: 22292002] 
31. Bertella L, Mori I, Grugni G, et al. Quality of life and psychological well-being in GH-treated, adult PWS patients: a longitudinal study. J Intellect Disabil Res. 2007; 51:302-311. [PubMed: 17326811]

32. Brown, H.; Prescott R, R. Applied mixed models in medicine. 2nd edition. Oxford, England: John Wiley \& Sons; 2006. p. 1-476.

33. Sanchez-Ortiga R, Klibanski A, Tritos NA. Effects of recombinant human growth hormone therapy in adults with Prader-Willi syndrome: a meta-analysis. Clin Endocrinol (Oxf). 2012; 77(1):86-93. [PubMed: 22117629]

34. Myers SE, Whitman BY, Carrel AL, Moerchen V, Bekx MT, Allen DB. Two years of growth hormone therapy in young children with Prader-Willi syndrome: physical and neurodevelopmental benefits. Am J Med Genet A. 2007; 143(5):443-448. [PubMed: 17103437]

35. Carrel AL, Myers SE, Whitman BY, Eickhoff J, Allen DB. Long-term growth hormone therapy changes the natural history of body composition and motor function in children with prader-willi syndrome. J Clin Endocrinol Metab. 2010; 95(3):1131-1136. [PubMed: 20061431]

36. Siemensma EP, Tummers-de Lind van Wijngaarden RF, Festen DA, Troeman ZC, van Alfen-van der Velden AA, Otten BJ, Hokken-Koelega AC. Beneficial effects of growth hormone treatment on cognition in children with Prader-Willi syndrome: a randomized controlled trial and longitudinal study. J Clin Endocrinol Metab. 2012; 97(7):2307-2314. [PubMed: 22508707]

37. Sode-Carlsen R, Farholt S, Rabben KF, et al. One year of growth hormone treatment in adults with Prader-Willi syndrome improves body composition: results from a randomized, placebo-controlled study. J. Clin. Endocrinol. Metab. 2010; 95:4943-4950. [PubMed: 20702523]

38. Sode-Carlsen R, Farholt S, Rabben KF, et al. Body composition, endocrine and metabolic profiles in adults with Prader-Willi syndrome. Growth Horm. IGF Res. 2010; 20:179-184. [PubMed: 20199883]

39. Sode-Carlsen R, Farholt S, Rabben KF, et al. Growth hormone treatment in adults with PraderWilli syndrome: the Scandinavian study. Endocr. 2012; 41:191-199.

40. Angulo MA, Castro-Magana M, Lamerson M, et al. Final adult height in children with PraderWilli syndrome with and without human growth hormone treatment. Am. J. Med. Genet. A. 2007; 143:1456-1461. [PubMed: 17567883]

41. Höybye C. Endocrine and metabolic aspects of adult Prader-Willi syndrome with special emphasis on the effect of growth hormone treatment. Growth Horm. IGF Res. 2004; 14:1-15. [PubMed: 14700552]

42. Eha, M. Organ tissue contribution to metabokicr rate. In: Kinney, JM.; Tucker, NH., editors. Energy Metabolism, Tissue determinants and cellular and corrolaries. New York: Raven Press; 1992. p. 61-77.

43. Sparti A, DeLany JP, de la Bretonne JA, Sander GE, Bray GA. Relationship between resting metabolic rate and the composition of the fat-free mass. Metabolism. 1997; 46(10):1225-1230. [PubMed: 9322812]

44. Matthews DR, Hosker JP, Rudenski AS, Naylor BA, Treacher DF, Turner RC. Homeostasis model assessment: insulin resistance and beta-cell function from fasting plasma glucose and insulin concentrations in man. Diabetologia. 1985; 28(7):412-419. [PubMed: 3899825] 

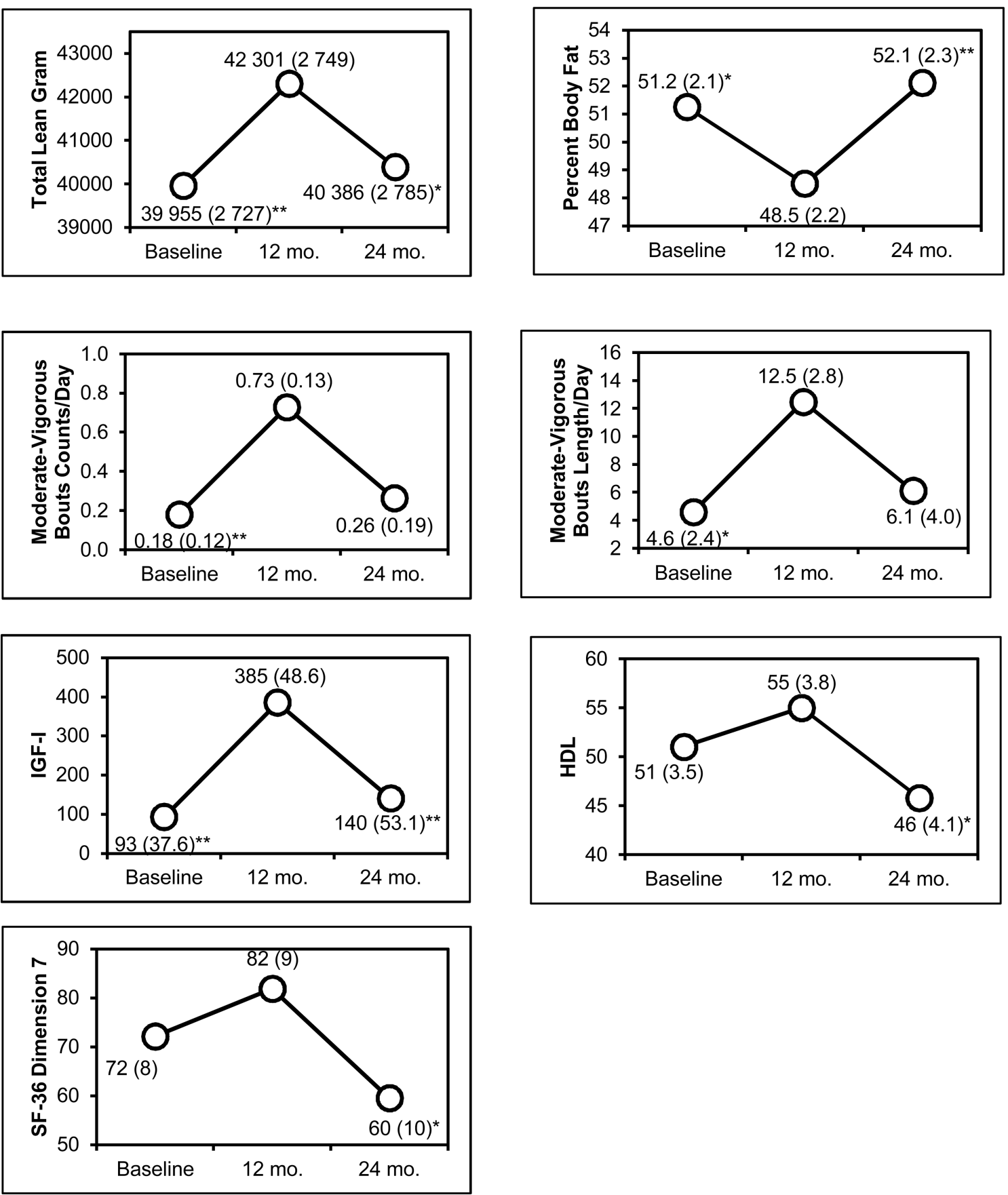

Figure 1.

Multiple Parameters Showing Significant Differences in Individuals with Prader-Willi Syndrome Receiving Growth Hormone Treatment $* \mathrm{p}<0.05 ; * \mathrm{*} p<0.01$ for fixed effect within-subject (time) comparison using 12 months as a reference point. 


\section{Table 1}

Baseline Descriptive Characteristics of Individuals with Prader-Willi Syndrome Grouped by Gender

\begin{tabular}{|l|c|c|}
\hline Variable & Females $(\mathbf{N}=\mathbf{6})$ & Males $(\mathbf{N}=\mathbf{5})$ \\
\hline Age $(\mathrm{yrs})$ & $33.8 \pm 12.2$ & $30.4 \pm 10.9$ \\
\hline Height $(\mathrm{cm})^{* *}$ & $144.7 \pm 1.6$ & $154.2 \pm 2.5$ \\
\hline Weight $(\mathrm{kg})^{* *}$ & $72.5 \pm 5.4$ & $98.9 \pm 7.2$ \\
\hline BMI & $34.5 \pm 2.2$ & $41.6 \pm 2.9$ \\
\hline Percent Body Fat & $51.0 \pm 2.3$ & $51.5 \pm 2.9$ \\
\hline $24 \mathrm{hr} \mathrm{EE} \mathrm{(kcal)}$ & $1929 \pm 124$ & $2654 \pm 274$ \\
\hline
\end{tabular}

$\mathrm{BMI}=$ Body mass index. EE=Energy expenditure. Values are means and standard errors. Independent samples t-test was used for comparison; p $<0.05$

$* *$

$\mathrm{p}<0.01$. 


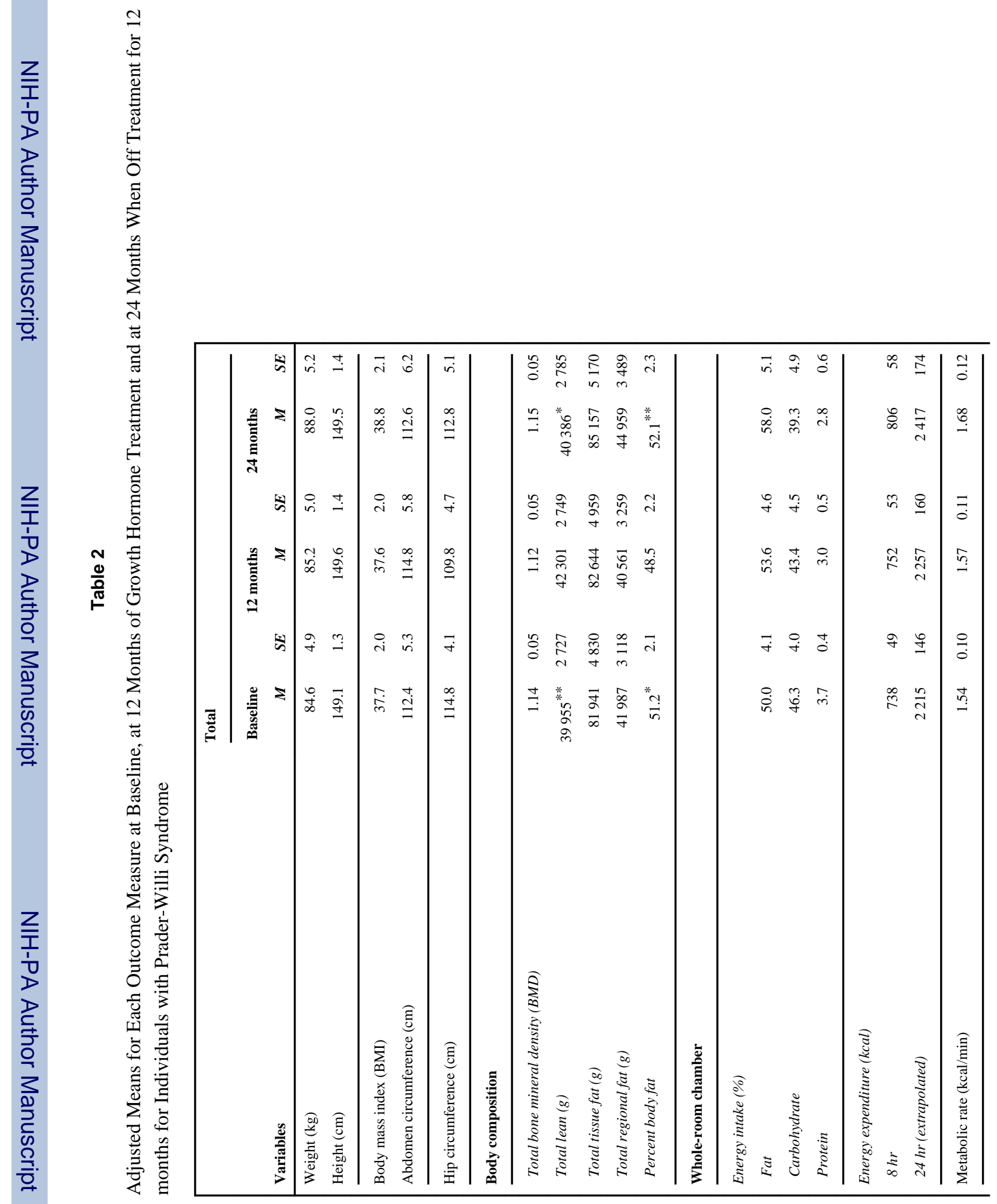




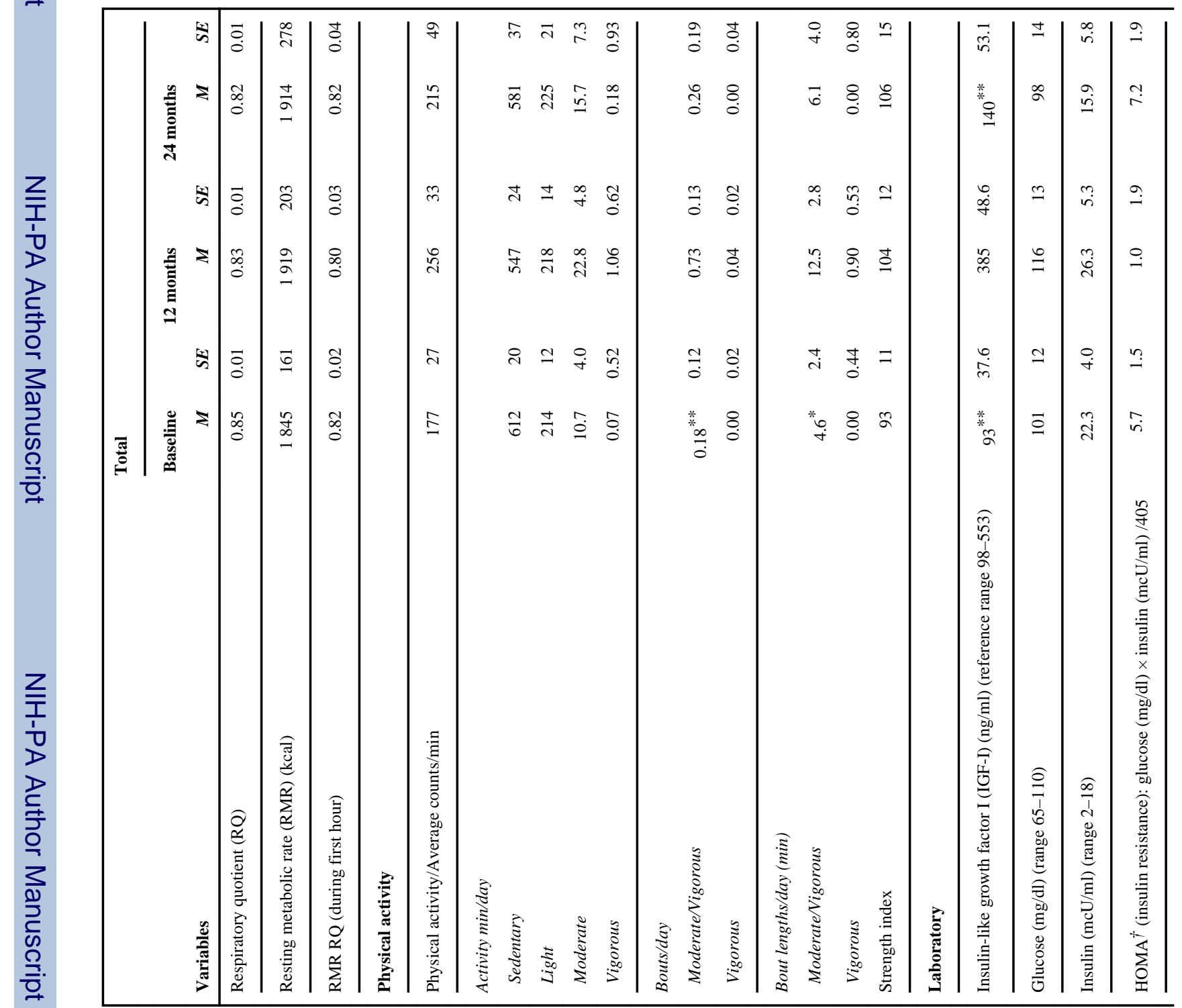




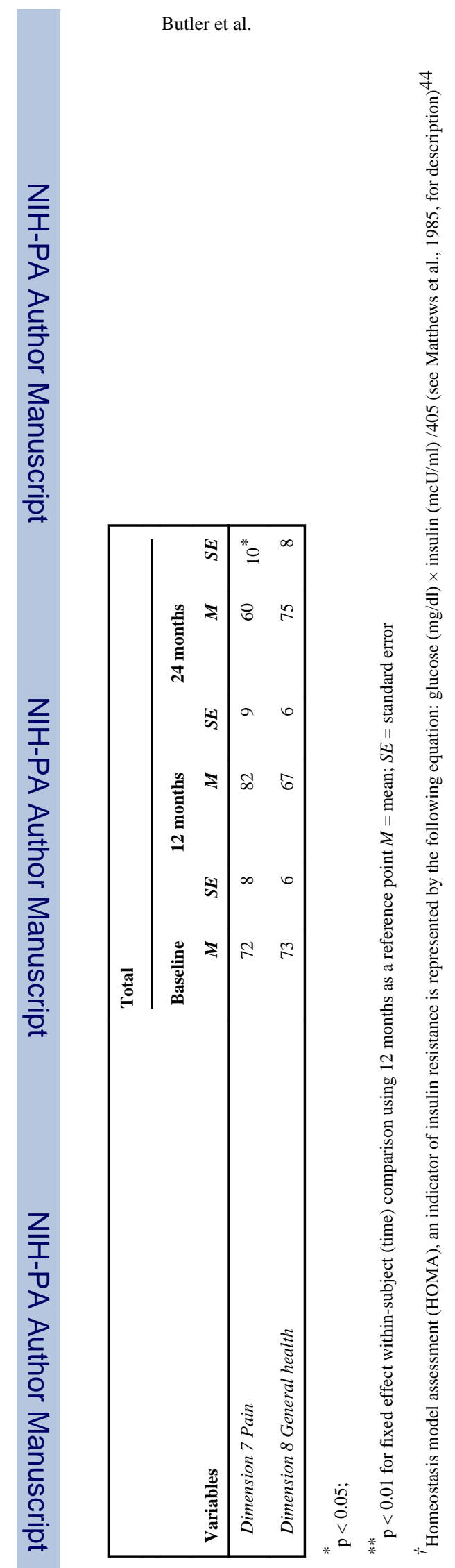

Page 18

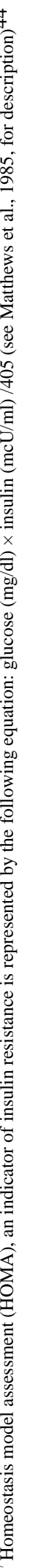

